\title{
Determinantes da Taxa de Imposto Efetiva de Empresas da América Latina
}

\author{
Determinants of Effective Tax Rate of Companies in Latin America
}

\author{
Camila Freitas Sant'Ana \\ Bacharel em Ciências Contábeis \\ Mestranda em Ciências Contábeis pela Universidade Regional de Blumenau - FURB \\ Rua Antônio da Veiga, 140 - Victor Konder - Blumenau, SC \\ CEP: 89012-900 \\ E-mail: camila-fsantana@ hotmail.com \\ Fone: (47) 3321-0565
}

\author{
Vinícius Costa da Silva Zonatto \\ Doutor em Contabilidade \\ Professor do Programa de Pós-Graduação em Ciências Contábeis \\ da Universidade Regional de Blumenau - FURB \\ Rua Antônio da Veiga, 140 - Victor Konder - Blumenau, SC \\ CEP: 89012-900 \\ E-mail: viniciuszonatto@gmail.com
}

Fone: (47) 3321-0565

\begin{abstract}
Resumo
O objetivo do estudo foi de identificar os fatores determinantes da taxa de imposto efetiva (ETR) de empresas da América Latina no período de 2009 à 2013. Estudo descritivo foi realizado por meio de pesquisa documental, com abordagem quantitativa dos dados. A amostra foi composta por 500 empresas, sendo 45 da Argentina, 171 do Brasil, 108 do Chile, 38 da Colômbia, 71 do México e 67 do Peru, cujos dados foram coletados na base de dados da Thompson Reuters ${ }^{\circledR}$ e analisados por meio de regressão de dados em painel através do software STATA ${ }^{\circledR}$, tendo como variável dependente a Taxa de Imposto Efetiva (ETR) e variáveis independentes o tamanho (TAM), a intensidade de capital (INTCAP), a intensidade de inventário (INTINV), a alavancagem (ALAV) e a rentabilidade dos ativos (ROA). Os resultados evidenciam que o tamanho das empresas apresenta influência positivamente significativa sobre a ETR das empresas colombianas. A intensidade de capital (INTCAP) e a intensidade de inventário (INTINV) não foram significativas para determinar a influência sobre a ETR nos países analisados. A alavancagem das empresas argentinas evidencia uma influência positiva sobre a ETR, enquanto que para as empresas colombianas esta influência foi negativamente significativa e não significativa para os demais países. A rentabilidade resultou em uma influência negativa para as empresas mexicanas e peruanas, e não significante para os demais países para determinar a influência sobre a ETR. Denota-se que existem diferenças quanto aos determinantes da carga fiscal em países latino-americanos, o que estimula a realização de novos estudos.
\end{abstract}

Palavras-chave: Taxa de Imposto Efetiva; Carga tributária; América Latina. 


\begin{abstract}
The objective of this study was to identify the determining factors of the effective tax rate (ETR) of companies of Latin America in the period 2009 to 2013. Descriptive study was conduct through documentary research, with a quantitative approach of the data. The sample was made up of 500 companies, being 45 of Argentina, 171 of Brazil, 108 of Chile, 38 of Colombia, and Mexico's 7167 of Peru. Whose data were collected in the database of Thompson Reuters ${ }^{\circledR}$ and analyzed by means of panel data regression through the software STATA ${ }^{\circledR}$, having as dependent variable the Effective tax rate (ETR) and independent variables size (TAM) capital intensity (INTCAP), the intensity of inventory (INTINV), leverage (ALAV) and profitability on assets (ROA). The results show that the size of companies positively influence presents significant about the ETR of Colombian companies. Capital intensity (INTCAP) and the intensity of inventory (INTINV) were not meaningful to determine the influence of the ETR in the countries analyzed. The leverage of the Argentine companies reveals a positive influence on the ETR, while for the Colombian companies this influence was significant not significant and negatively to the other countries. Profitability resulted in a negative influence to the Mexican and Peruvian companies, and not significant for the other countries to determine the influence on ETR. Denotes that there are differences regarding the determinants of tax burden in Latin American countries, which encourages further studies.
\end{abstract}

Keywords: Effective tax rate; Tax burden; Latin America.

\title{
1 Introdução
}

$\mathrm{Na}$ área de economia e finanças historicamente profissionais contábeis, especialistas em planejamento fiscal e analistas fiscais discutem na investigação fiscal a questão dos impostos, da legislação tributária e dos sistemas de tributação no âmbito da eficácia da gestão de negócios (SHACKELFORD; SHEVLIN, 2001; LAMMERSEN, 2002).

Cada País apresenta suas próprias normas e leis tributárias, bem como suas próprias peculiaridades no sentido de tributação do imposto de renda. De acordo com Caldeira (2006), a política fiscal afeta diretamente a competitividade das organizações, com influência significativa na formação do preço de venda que refletirá na liquidez e na rentabilidade das companhias.

Neste contexto, destaca-se que a carga tributária pode ocasionar em perda da competitividade das organizações, exigindo com que as empresas adotem alternativas para compensar economicamente seus investidores (ESNOLDE et al. 2009), como o estabelecimento de um planejamento tributário adequado (SANTOS; OLIVEIRA, 2008). Conforme explicam Formigoni, Antunes e Paulo (2009), o gerenciamento tributário oferece aos gestores a possibilidade de julgamento no processo de mensuração e evidenciação do resultado, considerando o atendimento das normas contábeis e da legislação tributária.

A mensuração do gerenciamento tributário pode ser realizada por meio da Taxa de Imposto Efetiva (Effective Tax Rates - ETR), que considera a taxa real de tributos incidentes sobre o lucro das empresas reportado ao mercado global, tratada como uma medida de avaliação da carga tributária e seu impacto sobre a atividade econômica (GIANNINI; MAGGIULLI, 2002).

Estudos desenvolvidos sobre a abordagem da Taxa de Imposto Efetiva centraram-se na investigação da alíquota efetiva da ETR resultante da prática de gerenciamento tributário (SHEVLIN, 1999; SHACKELFORD; SHEVLIN, 2001; MINNICK; NOGA, 2010; UTZIG et 
al., 2014). Alguns estudos diferem-se avaliando comparativamente a temática em diferentes países, procurando analisar os seus determinantes. Nesta perspectiva, Markle e Shackelford (2011) analisaram comparativamente a ETR em 78 países. Já Fernández-Rodríguez e Martínez-Arias (2014) identificaram as determinantes da ETR nas empresas dos países do BRIC. Os resultados encontrados nestes estudos são divergentes, estimulando a realização de novas pesquisas.

Conforme explicam Fernández-Rodríguez e Martínez-Arias (2014), não há consenso sobre as principais variáveis que explicam a ETR (tamanho, intensidade de capital e inventário, alavancagem e rentabilidade da empresa), devido ao fato dos resultados encontrados até este momento não serem conclusivos. Evidencia-se diferenças significativas entre os países, até mesmo dentro de seu contexto econômico interno.

Apesar de diversos estudos terem sido realizados sob a temática em diferentes contextos, pouco se sabe sobre as relações existentes entre a Taxa de Imposto Efetiva e seus determinantes em empresas da América Latina. Na última década as economias emergentes cresceram mais rapidamente do que as economias desenvolvidas, desempenhando um papel importante para a economia mundial. Os países latino-americanos compartilham certas características relacionadas a dimensão econômica e ao potencial de desenvolvimento (FERNÁNDEZ-RODRÍGUEZ; MARTÍNEZ-ARIAS, 2014). De acordo com Cano (2009), existem semelhanças em relação as pautas exportadoras, constituídas principalmente por produtos primários ou semimanufaturados nos países latino-americanos.

Neste contexto, considerando-se os resultados inconclusivos identificados na literatura, bem como há escassez de estudos desenvolvidos sob esta configuração na América Latina, elaborou-se a seguinte questão de pesquisa: quais são os fatores determinantes da taxa de imposto efetiva (ETR) de empresas da América Latina? Assim, para atingir a proposta de estudo tem-se o objetivo de verificar os fatores determinantes da taxa de imposto efetiva (ETR) de empresas da América Latina.

O estudo justifica-se por fornecer evidências sobre os determinantes da taxa de imposto efetiva (ETR) causada pela carga tributária fiscal incidente nas atividades de companhias abertas na Argentina, Brasil, Chile, Colômbia, México e Peru, sob o qual o relatório de Estatísticas sobre Receitas na América Latina, que analisa a evolução das receitas tributárias nos países latino-americanos, destaca que a arrecadação de impostos tem crescido, enquanto isso não é evidenciado nos países que fazem parte da Organização de Cooperação e de Desenvolvimento Econômico (OCDE) (CAMPOS, 2014).

Adicionalmente, a pesquisa justifica-se em razão da relevância do tema e sua contribuição para a literatura sobre a ETR, analisando sob perspectiva econômica os países da América Latina, contexto em que não foram encontrados estudos anteriores desenvolvidos sob a configuração proposta nesta pesquisa. O estudo abrange um período de análise de 2009 a 2013. A seleção deste período de análise é convergente as recomendações estabelecidas por Assaf Neto (2012), que descreve o período compreendido de 3 a 5 anos como necessário para a realização de uma análise de comparação temporal, possibilitando o acompanhamento na evolução dos indicadores estudados.

Além desta seção introdutória, que destaca a contextualização inicial apresentada para a realização desta pesquisa, apresenta-se na sequência o referencial teórico que fornece o embasamento teórico necessário a investigação. A seguir, descrevem-se os procedimentos metodológicos adotados para a realização da pesquisa. Por fim, efetua-se a apresentação da descrição, análise e discussão dos resultados, seguidos das considerações finais e das referências bibliográficas utilizadas para a realização do estudo. 


\section{Fundamentação Teórica}

Nesta seção apresenta-se a fundamentação teórica da pesquisa. Inicialmente são apresentadas as discussões gerais acerca do gerenciamento tributário e da taxa de imposto efetiva (ETR). Na sequência, são abordados os aspectos relacionados aos determinantes da taxa de imposto efetiva (ETR), que correspondem as variáveis da pesquisa, bem como os estudos que oferecem embasamento teórico a temática. Para a identificação dos estudos anteriores desenvolvidos sob a temática, utilizou-se as bases de busca dos dados bibliográficos Document Search (Scopus), Jstor, Portal de Periódicos Capes, Science Direct e Scientific Periodicals Electronic Library (SPELL).

\subsection{Gerenciamento tributário e Taxa de Imposto Efetiva (ETR)}

O gerenciamento de resultados tributários deriva da necessidade das empresas em diminuir o impacto econômico dos impostos nas contas de resultados, principalmente no que tange ao lucro das organizações. O gerenciamento tributário pode influenciar no montante atribuído à distribuição de dividendos e nos resultados reportados aos stakeholders e shareholders (PAULO; MARTINS; CORRAR, 2007).

Mesmo podendo ocasionar mudanças nos resultados reportados para o mercado, Formigoni, Antunes e Paulo (2009) salientam que o gerenciamento tributário não deve ser confundido como uma prática relacionada a fraude, em virtude que sua característica fundamental é a prática de gerenciamento de acordo com as normas contábeis e com a legislação tributária, do qual através do processo de mensuração e evidenciação pode surtir em alternativas válidas para reportar o resultado gerado pela organização.

$\mathrm{O}$ gerenciamento tributário pode ser definido como um procedimento legal que estabelece a possibilidade de estudo de alternativas que propiciem a redução da carga tributária das organizações (TANG, 2005; FORMIGONI; ANTUNES; PAULO, 2009; MINNICK; NOGA, 2010). Assim, o gerenciamento tributário é estabelecido por meio de um planejamento minucioso da adoção das práticas legais de tributação com o objetivo de redução da carga tributária (TANG, 2005).

No decorrer dos últimos anos as reformas fiscais promoveram o aumento do interesse quanto à avaliação dos efeitos da tributação em relação ao desempenho econômico das organizações. O processo de globalização da economia mundial desencadeou uma pressão sobre os governos para tornar os países mais atraentes para investidores e assim assegurar as políticas econômicas de desenvolvimento (CAREY; RABESONA, 2002).

Nesta perspectiva, conforme explicam Shackelford e Shevlin (2001), a ETR é vista como um indicador que auxilia os tomadores de decisão a identificar o real valor desembolsado pelas organizações no que tange ao volume de tributos recolhidos sobre o lucro, permitindo-se confrontar a carga real efetiva praticada pela empresa com a carga estabelecida pela legislação tributária.

De acordo com Utzig et al. (2014), as empresas apresentam dificuldades em analisar efeitos da tributação, em virtude da falta de medidas de gerenciamento adequadas para realizar a tributação. Segundo Shackelford e Shevlin (2001), a preocupação dos gestores em relação à questão fiscal volta-se em coordenar os fatores tributários que influenciam no processo de tomada de decisão, pois estes podem promover impactos sobre os objetivos da companhia.

Neste sentido, denota-se que a ETR destaca a influência da carga de tributação sobre o projeto de investimento (KNIRSCH, 2002). Desta forma, por meio da utilização da ETR, torna-se possível a identificação de possíveis distorções nas alíquotas de imposto praticadas em determinados mercados (SHEVLIN, 1999), bem como auxiliar os gestores no 
estabelecimento de estratégias de gestão fiscal para o planejamento tributário futuro (MINNICK; NOGA, 2010).

A este respeito, Giannini e Maggiulli (2002) discorrem que para avaliar o impacto e compreender os efeitos da tributação é primordial confrontar-se o resultado da ETR com as alíquotas previstas em lei, em virtude de que desta forma obter-se-á o real impacto fiscal sofrido intrinsicamente nas atividades desenvolvidas pela empresa.

$\mathrm{Na}$ literatura revisitada sobre a temática, encontram-se estudos que apontam para a existência de fatores determinantes da ETR, que é obtida através da razão entre a apuração do imposto de renda e o lucro contábil antes dos impostos (LAIR), do qual pode-se visivelmente identificar a presença do gerenciamento quando denota-se a situação em que duas organizações apresentam mesmo lucro, porém montantes de desembolsos de imposto de renda diferentes (REGO, 2003). Entretanto, não há consenso na literatura sobre os determinantes da ETR entre organizações de diferentes países, o que estimula a realização de novos estudos (FERNÁNDEZ-RODRÍGUEZ; MARTÍNEZ-ARIAS, 2014).

Apesar de tais evidências, deve-se considerar as possíveis variáveis explicativas da ETR registrada pelas organizações, uma vez que o processo de tomada de decisões também pode ser influenciado pelas repercussões fiscais, advindas das decisões de investimento e financiamento de capital (FERNÁNDEZ-RODRÍGUEZ; MARTÍNEZ-ARIAS, 2014). Assim, no próximo tópico efetua-se a apresentação das variáveis encontradas na literatura como determinantes da taxa de imposto efetiva e os estudos que embasam a sustentação das hipóteses elaboradas para a realização desta pesquisa.

\subsection{Determinantes da Taxa de Imposto Efetiva (ETR) e Hipóteses da pesquisa}

Vários estudos objetivaram analisar as ETRs em contextos diversos. Nas pesquisas desenvolvidas por Zimmerman (1983) e Wang (1991) foi evidenciado que o tamanho da empresa influencia positivamente a taxa de imposto efetiva e os custos políticos. Ainda nesta perspectiva, o estudo de Omer, Molloy e Ziebart (1993) investigou a relação entre o tamanho da empresa e encargos fiscais, sob o qual os resultados indicam que a amostra apresenta diferenças significativamente positivas entre o tamanho da empresa e a carga fiscal das organizações.

Entretanto, a partir dos resultados encontrados na pesquisa desenvolvida por Gupta e Newberry (1997), aplicada na Espanha observando as mudanças ocasionadas antes e pós a obrigatoriedade da Lei da Reforma Tributária instituída no ano de 1986, contemplando o período anterior de 1982 a 1985 e o período posterior de 1987 a 1990 da adoção da referida lei. Os autores identificaram que a ETR não está associada com o tamanho da empresa, porém com outras variáveis. Segundo estes autores, as variáveis que compreendem a estrutura de capital de uma empresa, o mix de ativos e sua performance estão positivamente associadas a ETR. Os resultados encontrados pelos autores lançam dúvidas em relação à reforma aplicada no ano de análise, assim como os achados de Wilkinson, Cahan e Jones (2001), que incluíram em suas análises o lucro da empresa e a distribuição de dividendos para verificar se estas varáveis causaria diminuição da ETR, sob o qual identificaram que os dividendos pagos aos acionistas eram tributados a uma taxa maior do que os lucros não distribuídos, não ocasionando em redução da taxa de imposto efetiva, de modo que quanto maior os dividendos distribuídos sobre o lucro da empresa maior a ETR.

A pesquisa realizada por Kim e Limpaphayom (1998) analisou a relação entre as taxas de imposto efetiva e tamanho das empresas dos países de Hong Kong, Coréia, Malásia, Taiwan e Tailândia, por meio da aplicação de regressão linear, cujos resultados evidenciaram uma relação negativa entre o tamanho da empresa e as taxas de imposto efetivas nas economias emergentes do pacífico. Nesta mesma perspectiva de análise, o estudo de Derashid e Zhang (2003) identificou no contexto das empresas da Malásia no período de 1990 a 1999 
por meio da aplicação de regressão linear, que as grandes empresas da Malásia não sofrem com um custo político, fato constatado pela relação negativamente significativa confirmada entre o tamanho da empresa e a ETR.

No estudo de Harris e Feeny (2003), os resultados encontrados pelos autores indicaram que o tamanho da empresa, o nível de alavancagem, a intensidade de capital, a receita líquida e os investimentos em $\mathrm{P} \& \mathrm{D}$ são variáveis importantes para a determinação da taxa de imposto efetiva das organizações. Plesko (2003) também analisou as variáveis que produzem efeitos sobre a carga fiscal das organizações, considerando as variáveis intensidade de capital, intensidade de inventário e alavancagem. As evidências encontradas pelo autor permitiram concluir que estas proxies fornecem coeficientes capazes de determinar os efeitos na tributação.

Por sua vez, Janssen (2005) evidenciou em uma amostra de empresas holandesas, no período de 1994 a 1999, que a ETR não difere muito das alíquotas legais daquele País. Seus achados indicam que a intensidade de capital está negativamente associada com a ETR. Já Feeny, Gillman e Harris (2005) aplicando estudo similar no contexto das empresas australianas, encontraram resultados que apontam como determinantes da ETR as despesas com juros, amortizações, o endividamento, a estruturas de ativos e a propriedade estrangeira das empresas.

Na pesquisa de Calvé, Labatut e Molina (2005), os autores analisaram o impacto causado pela reforma tributária ocorrida em 1995 sobre a criação de um regime especial de tributação para as pequenas empresas, como forma de verificar a carga fiscal suportada pelas pequenas empresas de Valência e quais foram as variáveis econômicas que apresentaram maior poder explicativo em relação a taxa de imposto efetiva no período de 1992-1999. Por meio de análise fatorial os autores constataram que as pequenas empresas, obtendo benefícios fiscais, diminuíram a taxa de imposto efetiva e que há uma relação positivamente significativa com a rentabilidade, o tamanho da empresa, o grau de dependência financeira externa e uma relação negativamente significativa com o nível de investimento em ativos fixos, concluindo que empresas com maior rentabilidade e tamanho dos ativos suportam maiores pressões fiscais, enquanto que empresas com maior grau de endividamento e ativos imobilizados conseguem reduzir sua ETR.

Richardson e Lanis (2007) em seu estudo também encontraram evidências de que a ETR está associada ao tamanho da empresa, a estrutura de capital, a alavancagem, a intensidade de capital, a intensidade do inventário e a intensidade de P\&D. Contudo, no estudo de Liu e Cao (2007), em que foram examinadas as empresas da China, no período de 1998 a 2004, os autores encontraram evidências de que o tamanho da empresa e a intensidade de capital não obtiveram efeitos significativos sobre a ETR, entretanto a alavancagem demonstrou influência significativamente negativa com a ETR, que resultou ainda relacionada com as políticas de incentivo fiscal, assim como a rentabilidade e a estrutura de propriedade das empresas pesquisadas.

Na pesquisa de Chen et al. (2010) os achados encontrados por estes autores destacam que as empresas familiares sofrem com menos pressões fiscais, em virtude de que abrem mão de incentivos e benefícios fiscais para redução da ETR e por estarem mais preocupadas com a penalidade e a reputação de danos decorrentes de uma auditoria, do que quando confrontadas com empresas não familiares.

Recentemente Fernández-Rodríguez e Martinez-Arias (2011) realizaram uma comparação entre as ETRs e suas determinantes identificadas nas amostras das empresas dos Estados Unidos e da União Europeia (UE), no período de 1995 a 2007. Os resultados encontrados permitiram aos autores concluir que as empresas americanas apresentam menor ETR que as empresas da União Europeia, e que a ETR é determinada de acordo com as caraterísticas das empresas e as políticas fiscais adotadas pelo governo. No entanto, no estudo 
de Díaz, Fernandes-Rodrigues e Martinez-Arias (2011), em que foram analisadas as determinantes da ETR em uma amostra de empresas do setor bancário espanhol no período de 1993 a 2004, os resultados encontrados pelos autores evidenciaram que a ETR é menor que a carga fiscal e que as organizações maiores, com menos capital e níveis mais elevados de ativos fixos apresentam uma carga tributária menor, enquanto os bancos com maior rentabilidade apresentam ETR mais elevadas, revelando ainda que as determinantes da ETR variam de acordo com o tipo de entidade pesquisada.

Estes resultados são parcialmente corroborados com os achados encontrados no estudo desenvolvido por Fernández-Rodríguez e Martínez-Arias (2014). Segundo estes autores, as determinantes da ETR das empresas dos países do BRIC (Brasil, Rússia, Índia e China) durante o período de 2000 a 2009, dentre as variáveis tamanho, intensidade de capital, intensidade de inventário, alavancagem e rentabilidade da empresa, apenas a variável intensidade de inventário apresentou significância estatística em todos os países do BRIC, revelando uma influência positiva entre ETR e esta variável. As demais variáveis também afetam a ETR, no entanto, apresentam diferenças entre os países da análise realizada.

De maneira geral, como pode-se verificar a partir dos estudos anteriores encontrados na revisão da literatura, diferentes determinantes são utilizados para explicar a taxa de imposto efetiva (ETR) incidente nas empresas. Da mesma forma, os resultados encontrados para algumas destas variáveis também divergem, o que evidencia que em diferentes países tais variáveis podem apresentar relação diferente com a taxa de imposto efetiva (ETR).

Neste contexto, dentre os estudos apresentados nesta pesquisa, destaca-se a utilização da variável "tamanho da empresa", medida pelo logaritmo do ativo total, como a principal determinante da taxa de imposto efetiva (ETR). Conforme explicam Fernández-Rodríguez e Martínez-Arias (2014), o uso da variável tamanho se estabelece sobre duas razões distintas, sendo a primeira relacionada positivamente aos custos políticos, sob o qual as maiores empresas expõem-se a maiores ações regulamentadas, e a segunda relacionada negativamente as maiores empresas, que apresentam melhor planejamento tributário para a diminuição da ETR, podendo ainda desfrutar do poder político.

A este respeito, nos estudos de Kim e Limpaphayom (1998), Derashid e Zhang (2003), Harris e Feeny (2003), Janssen (2005) e Richardson e Lanis (2007) foram encontradas evidências sugerindo uma influência negativa entre o tamanho da empresa e a taxa de imposto efetiva. Já nos estudos desenvolvidos por Zimmerman (1983), Wang (1991), Omer, Molloy e Ziebart (1993), Calvé, Labatut e Molina (2005) e Chen et al. (2010) evidenciou-se uma influência positiva entre o tamanho da empresa e a ETR. Contudo, as pesquisas desenvolvidas por Gupta e Newberry (1997), Wilkinson, Cahan e Jones (2001) e Liu e Cao (2007) não apresentaram significância entre o tamanho da empresa e a ETR. Tendo em vista os resultados conflitantes encontrados na literatura e considerando-se os achados de Fernández-Rodríguez e Martinez-Arias (2011) e de Díaz, Fernandes-Rodrigues e Martinez-Arias (2011), que evidenciaram uma influência positiva não-linear entre as variáveis tamanho e taxa de imposto efetiva, indicando que a carga tributária das grandes empresas torna-se menor em determinado momento, estabeleceu-se a seguinte hipótese de pesquisa:

\section{$\mathrm{H}_{1}$ - Existe uma influência positiva entre o tamanho e a taxa de imposto efetiva.}

Em relação a variável que representa a intensidade de capital, os estudos de Gupta e Newberry (1997), Derashid e Zhang (2003), Calvé, Labatut e Molina (2005), Janssen (2005), Chen et al. (2010) e Díaz, Fernandes-Rodrigues e Martinez-Arias (2011) encontraram em seus achados uma influência negativa, indicando que quanto maior o peso do imobilizado corpóreo menor será a ETR. Entretanto, os achados de Plesko (2003) e de Feeny, Gillman e Harris (2005) encontraram uma relação direta entre a intensidade de capital e a carga fiscal, resultado 
que foi evidenciado de forma significativamente positiva no estudo de Fernández-Rodríguez e Martínez-Arias (2011), o qual serviu de base para a determinação da seguinte hipótese de pesquisa:

\section{$\mathbf{H}_{2}$ - Existe uma influência positiva entre a intensidade de capital e a taxa de imposto efetiva.}

No que refere-se a intensidade de inventário, de acordo com o estudo de FernándezRodríguez e Martínez-Arias (2014), o mix de ativos, compostos pelo imobilizado corpóreo, apresentam uma influência sobre o ETR das empresas, em virtude de que o gerenciamento das depreciações da propriedade, planta e equipamento são utilizados para deduzir a ETR. Assim, o autor denota que a possibilidade de obter menores ETRs dependerá do volume de ativos correntes das empresas. Partindo-se dessa premissa, considera-se que quanto maior o nível de inventário maior será a carga fiscal da organização. Assim, formulou-se a seguinte hipótese:

\section{$\mathrm{H}_{3}$ - Existe uma influência positiva entre a intensidade de inventário e a taxa de imposto efetiva.}

Quanto a alavancagem, os estudos de Plesko (2003), Calvé, Labatut e Molina (2005), Liu e Cao (2007) e de Richardson e Lanis (2007) em seus achados encontram uma influência negativamente significativa entre a alavancagem e a ETR. Porém, não foi evidenciado nenhuma influência significativa na pesquisa desenvolvida por Kim e Limpaphayom (1998) e Wilkinson, Cahan e Jones (2001). Entretanto, os estudos desenvolvidos por Harris e Feeny (2003), Janssen (2005) e Chen et al. (2010) evidenciaram uma influência positiva entre a alavancagem e a ETR, assim como também foi evidenciado no estudo de FernándezRodríguez e Martínez-Arias (2011), porém, ressaltando-se que até certo nível esta influência é positiva e depois passa a apresentar-se de forma negativa. Desta forma, os achados enfatizam que a medida que as empresas buscam aumentar sua alavancagem as mesmas estão mais propensas a assumir dívidas e assim consequentemente reduzir suas ETR, já que o valor é deduzido da base de tributação. Partindo desta concepção, destaca-se a seguinte hipótese: efetiva.

\section{$H_{4}$ - Existe uma influência positiva entre a alavancagem e a taxa de imposto}

Em se tratando da rentabilidade medida pelo retorno dos ativos (ROA), na literatura se destaca que esta variável é um fator determinante para a carga fiscal. Quanto maior a rentabilidade maior a ETR. Destaca-se que esta influência positiva foi encontrada nos estudos de Gupta e Newberry (1997), Plesko (2003), Calvé, Labatut e Molina (2005), Richardson e Lanis (2007), Chen et al. (2010) e de Fernández-Rodríguez e Martínez-Arias (2011). No entanto, o estudo de Derashid e Zhang (2003) vai de encontro a estes resultados, uma vez que os achados no contexto das empresas da Malásia apontaram para uma influência negativa entre a rentabilidade e a ETR, em que as empresas com maior rentabilidade apresentaram uma menor carga tributária. Já no estudo de Díaz, Fernandes-Rodrigues e Martinez-Arias (2011), a influência de efeito encontrada não foi significativa entre a rentabilidade e a ETR. Tendo em vista os resultados encontrados na maioria dos estudos revisitados, para a realização desta pesquisa estabeleceu-se a seguinte hipótese: efetiva.

$$
H_{5} \text { - Existe uma influência positiva entre a rentabilidade e a taxa de imposto }
$$


Definidas as hipóteses a serem testadas na pesquisa, a seguir descreve-se os procedimentos metodológicos utilizados para a realização do estudo.

\section{Procedimentos Metodológicos}

Com o intuito de atender ao objetivo de pesquisa de identificar os fatores determinantes da taxa de imposto efetiva (ETR) de empresas da América Latina, realizou-se uma pesquisa descritiva, realizada por meio de análise documental e abordagem quantitativa dos dados.

A população da pesquisa compreende todas as empresas listadas nas bolsas de valores dos respectivos países, Argentina, Brasil, Chile, Colômbia, México e Peru, que totalizam 1.266 empresas de capital aberto. Entretanto, para composição da amostra da pesquisa, foram excluídas da amostra empresas financeiras e de seguros resultando em uma amostra inicial de 847 empresas, do qual ainda foram excluídas as empresas que não continham todas as informações necessárias para a análise dos dados no período objeto de investigação, o que resultou em uma amostra final investigada de 500 empresas, cuja distribuição por País é apresentada na Tabela 1.

Tabela 1 - Distribuição da amostra por países

\begin{tabular}{c|c|c|c|c|c|c}
\hline \multirow{2}{*}{ País } & \multicolumn{2}{|c|}{ População } & \multicolumn{1}{c}{$\begin{array}{c}\text { Amostra inicial (população } \\
\text { menos financeiras) }\end{array}$} & \multicolumn{2}{c}{ Amostra final } \\
\cline { 2 - 7 } & Freq. Abs. & Freq. Rel. & Freq. Abs. & Freq. Rel. & Freq. Abs. & Freq. Rel. \\
\hline Argentina & 103 & $8.1 \%$ & 82 & $9.7 \%$ & 45 & $9.0 \%$ \\
\hline Brasil & 493 & $38.9 \%$ & 296 & $34.9 \%$ & 171 & $34.2 \%$ \\
\hline Chile & 229 & $18.1 \%$ & 174 & $20.5 \%$ & 108 & $21.6 \%$ \\
\hline Colômbia & 76 & $6.0 \%$ & 58 & $6.8 \%$ & 38 & $7.6 \%$ \\
\hline México & 151 & $11.9 \%$ & 105 & $12.4 \%$ & 71 & $14.2 \%$ \\
\hline Peru & 214 & $16.9 \%$ & 132 & $15.6 \%$ & 67 & $13.4 \%$ \\
\hline Total & $\mathbf{1 . 2 6 6}$ & $100.0 \%$ & $\mathbf{8 4 7}$ & $100.0 \%$ & $\mathbf{5 0 0}$ & $100.0 \%$ \\
\hline
\end{tabular}

Fonte: Dados da pesquisa.

O procedimento adotado para a coleta de dados ocorreu por meio de pesquisa documental, realizada a partir dos demonstrativos financeiros divulgados pelas empresas de capital aberto dos países da América Latina, disponíveis na base de dados Thomson Reuters ${ }^{\circledR}$, no período compreendido de 2009 a 2013. A partir da revisão da literatura realizada, estabeleceram-se as variáveis selecionadas para esta investigação, as quais são apresentadas no Quadro 1.

Quadro 1 - Variáveis analisadas

\begin{tabular}{|c|c|c|c|}
\hline Tipo & Variáveis & Descrição & Autores \\
\hline $\begin{array}{l}\stackrel{0}{0} \\
\frac{0}{0} \\
\stackrel{0}{0} \\
\stackrel{0}{0} \\
\stackrel{0}{0}\end{array}$ & ETR & $\begin{array}{l}\text { Taxa de Imposto } \\
\text { Efetivo (Provisão } \\
\text { p/ IR e CS / Lucro } \\
\text { antes do IR e CS) }\end{array}$ & $\begin{array}{l}\text { Zimmerman (1983); Wang (1991); Omer, Molloy e Ziebart (1993); } \\
\text { Gupta e Newberry (1997); Kim e Limpaphayom (1998); } \\
\text { Wilkinson, Cahan e Jones (2001); Derashid e Zhang (2003); Harris } \\
\text { e Feeny (2003); Calvé, Labatut e Molina (2005); Janssen (2005) e } \\
\text { Richardson e Lanis (2007); Liu e Cao (2007); Chen et al. (2010); } \\
\text { Fernández-Rodríguez e Martinez-Arias (2011); Díaz, Fernandes- } \\
\text { Rodrigues e Martinez-Arias (2011); Fernández-Rodríguez e } \\
\text { Martínez-Arias (2014). }\end{array}$ \\
\hline $\begin{array}{l}\frac{0}{0} \\
\frac{\tilde{D}}{0} \\
\frac{\tilde{D}}{0} \\
\Xi\end{array}$ & TAM & $\begin{array}{c}\text { Tamanho (Log do } \\
\text { Ativo Total) }\end{array}$ & $\begin{array}{l}\text { Zimmerman (1983); Wang (1991); Omer, Molloy e Ziebart (1993); } \\
\text { Gupta e Newberry (1997); Kim e Limpaphayom (1998); } \\
\text { Wilkinson, Cahan e Jones (2001); Derashid e Zhang (2003); Harris } \\
\text { e Feeny (2003); Calvé, Labatut e Molina (2005); Janssen (2005) e } \\
\text { Richardson e Lanis (2007); Liu e Cao (2007); Chen et al. (2010); }\end{array}$ \\
\hline
\end{tabular}




\begin{tabular}{|c|c|c|}
\hline & & $\begin{array}{l}\text { Fernández-Rodríguez e Martinez-Arias (2011); Díaz, Fernandes- } \\
\text { Rodrigues e Martinez-Arias (2011); Fernández-Rodríguez e } \\
\text { Martínez-Arias (2014). }\end{array}$ \\
\hline INTCAP & $\begin{array}{l}\text { Intensidade de } \\
\text { Capital (Ativos } \\
\text { fixos tangíveis / } \\
\text { Ativo total) }\end{array}$ & $\begin{array}{l}\text { Gupta e Newberry (1997); Derashid e Zhang (2003); Plesko } \\
\text { (2003); Calvé, Labatut e Molina (2005); Feeny, Gillman e Harris } \\
\text { (2005); Janssen (2005); Chen et al. (2010); Díaz, Fernandes- } \\
\text { Rodrigues e Martinez-Arias (2011); Fernández-Rodríguez e } \\
\text { Martínez-Arias (2011); Fernández-Rodríguez e Martínez-Arias } \\
\text { (2014). }\end{array}$ \\
\hline INTINV & $\begin{array}{l}\text { Intensidade do } \\
\text { Inventário } \\
\text { (Estoques / Ativo } \\
\text { total) }\end{array}$ & Fernández-Rodríguez e Martínez-Arias (2014) \\
\hline ALAV & $\begin{array}{l}\text { Alavancagem } \\
\text { (Dívidas de L.P. / } \\
\text { Patrimônio } \\
\text { Líquido) }\end{array}$ & $\begin{array}{l}\text { Kim e Limpaphayom (1998); Wilkinson, Cahan e Jones (2001); } \\
\text { Harris e Feeny (2003); Plesko (2003); Calvé, Labatut e Molina } \\
\text { (2005); Janssen (2005); Liu e Cao (2007); Richardson e Lanis } \\
\text { (2007); Chen et al. (2010); Fernández-Rodríguez e Martínez-Arias } \\
\text { (2011); Fernández-Rodríguez e Martínez-Arias (2014) }\end{array}$ \\
\hline ROA & $\begin{array}{l}\text { Rentabilidade } \\
\text { dos Ativos (Lucro } \\
\text { líquido / Ativo } \\
\text { Total) }\end{array}$ & $\begin{array}{l}\text { Gupta e Newberry (1997); Derashid e Zhang (2003); Plesko } \\
\text { (2003); Calvé, Labatut e Molina (2005); Richardson e Lanis } \\
\text { (2007); Chen et al. (2010); Fernández-Rodríguez e Martínez-Arias } \\
\text { (2011); Díaz, Fernandes-Rodrigues e Martinez-Arias (2011); } \\
\text { Fernández-Rodríguez e Martínez-Arias (2014) }\end{array}$ \\
\hline
\end{tabular}

Fonte: Dados da pesquisa.

Efetuada a coleta dos dados necessários a realização da pesquisa, a seguir foram calculadas as ETRs das empresas selecionadas, considerando-se os países de origem. A mensuração da taxa de imposto efetiva determinada sobre o lucro foi estabelecida utilizandose a fórmula destacada por Shevlin (1999):

$$
E T R=\frac{\text { Provisão para IR e CS }}{\text { Lucro Antes do IR e CS (LAIR) }}
$$

Ainda, conforme as variáveis observadas no Quadro 1, formulou-se uma equação para a análise de dados em painel, com base nos estudos que analisaram as determinantes da ETR, como o estudo de Fernández-Rodríguez e Martínez-Arias (2014). Desta forma, estabeleceu-se a seguinte equação:

$$
\operatorname{ETR}_{i t}=\varphi_{0}+\varphi_{1} \operatorname{TAM}_{i t}+\varphi_{2} \operatorname{INTCAP}_{i t}+\varphi_{3} \operatorname{INTINV}_{i t}+\varphi_{4} A L A V_{i t}+\varphi_{5} \operatorname{ROA}_{i t} \varepsilon_{i t}
$$

A presente fórmula apresenta como variável dependente de teste, a taxa de imposto efetiva (ETR), e como variáveis independentes o tamanho da empresa (TAM), a intensidade de capital (INTCAP), a intensidade de inventário (INTINV), a alavancagem financeira da empresa (ALAV) e a rentabilidade dos ativos (ROA).

Os dados coletados foram tabulados em planilhas eletrônicas, em que inicialmente foram calculadas as ETRs dos países e, posteriormente, realizada a comparação de média das taxas entre os países da América Latina e a aplicação da técnica de análise estatística multivariada de regressão de dados em painel, considerando os anos de 2009, 2010, 2011, 2012 e 2013, com o auxílio do software STATA®. Os resultados encontrados são apresentados a seguir. 


\section{Análise e discussão dos resultados}

Neste capítulo apresentam-se os resultados obtidos a partir da pesquisa realizada, em que os dados resultantes da ETR foram comparados entre os países analisados na amostra investigada na pesquisa, a partir da análise das médias das ETR. A Tabela 2 apresenta os resultados da taxa de imposto efetiva identificada em cada País.

Tabela 2 - Taxa de Imposto Efetiva por País

\begin{tabular}{c|c|c|c|c|c|c}
\hline País & ETR 2009 & ETR 2010 & ETR 2011 & ETR 2012 & ETR 2013 & Média Geral \\
\hline Argentina & $44.76 \%$ & $48.77 \%$ & $33.54 \%$ & $42.57 \%$ & $58.85 \%$ & $45,70 \%$ \\
\hline Brasil & $41.29 \%$ & $50.81 \%$ & $37.16 \%$ & $67.64 \%$ & $31.43 \%$ & $45.67 \%$ \\
\hline Chile & $20.64 \%$ & $23.82 \%$ & $32.68 \%$ & $23.16 \%$ & $47.23 \%$ & $29.51 \%$ \\
\hline Colômbia & $20.48 \%$ & $20.01 \%$ & $56.05 \%$ & $33.70 \%$ & $63.96 \%$ & $38.84 \%$ \\
\hline México & $36.58 \%$ & $72.67 \%$ & $30.73 \%$ & $50.26 \%$ & $31.53 \%$ & $44,36 \%$ \\
\hline Peru & $34.49 \%$ & $30.95 \%$ & $29.67 \%$ & $26.48 \%$ & $68.99 \%$ & $38.12 \%$ \\
\hline
\end{tabular}

Fonte: Dados da pesquisa.

Com base nos resultados apresentados na Tabela 2, observa-se que a menor média da taxa de imposto efetiva foi registrada pelas empresas chilenas com $29,51 \%$, considerando-se todo o período de 2009 a 2013. No entanto, observa-se uma variação ascendente das ETR apresentadas pelas empresas deste País, que evoluíram do período inicial analisado (2009) de uma taxa média de 20,48\% para 47,23\% no período de 2013.

Destaca-se que as maiores ETR médias registradas no período de 2009 a 2013 estão relacionadas as empresas argentinas, que apresentaram uma ETR média de 45,70\%. Em segundo lugar encontraram-se as taxas das empresas brasileiras com 45,67\%, seguidas em terceiro lugar pelas taxas identificadas junto as empresas mexicanas. Os resultados encontrados corroboram com o destaque de Campos (2014), que relatou que a Argentina lidera o ranking de carga tributária da América Latina com 37,3\%, seguida pelo Brasil com $36,3 \%$, conforme os dados registrados pela OCDE no ano de 2012, considerando os resultados de acordo com o Produto Interno Bruto - PIB de cada País latino-americano.

Neste contexto, os resultados evidenciados indicam que a carga tributária medida pela amostra investigada vem apresentando um aumento considerável na ETR no decorrer dos últimos 5 anos, resultado que corrobora com os achados de Campos (2014) que indicou que a carga tributária em geral vem crescendo nos países latino-americanos desde 2010.

Após a identificação da taxa de imposto efetiva média da amostra analisada em cada País, na sequência procurou-se verificar quais os fatores que influenciam os resultados obtidos em relação as ETRs em cada País. Os dados das variáveis apresentadas no Quadro 1 foram submetidos à análise estatística multivariada por meio da aplicação de regressão de dados em painel a partir do software STATA®.

Deste modo, de acordo com as exigências estatísticas recomendadas por Hair Jr. (2009), para se estabelecer a confiabilidade e validade dos dados utilizados para determinar o modelo empregado para a análise dos resultados da pesquisa, inicialmente submeteu-se os dados analisados a testes de homoscedasticidade, multicolinearidade, de ausência de autocorrelação dos resíduos e de normalidade. Os resultados encontrados alcançaram índices satisfatórios demonstrando que as variáveis não apresentam alta correlação e destacam uma distribuição normal dos dados, validando as variáveis utilizadas para análise dos dados.

Após a realização dos testes iniciais, foi aplicado para o estabelecimento da regressão de dados em painel o teste LM de Breusch-Pagan e de Hausman, que tem por objetivo indicar qual o modelo será utilizado para análise, sendo de Efeito Fixo ou de Efeito Aleatório. Os resultados encontrados indicaram a utilização do modelo de Efeitos Fixos para todos os países da amostra investigada (Argentina, Brasil, Chile, Colômbia, México e Peru), em virtude que os resultados do teste LM de Breusch-Pagan foi 0,000 demonstrando a aceitação da hipótese 
de que a variância dos resíduos que refletem as diferenças individuais é igual a zero e o teste de Hausman que foi 0,000, não demonstrando a utilização de efeitos aleatórios.

Os principais resultados da regressão de dados em painel apresentam-se sintetizados no Quadro 2 destacados de acordo com os modelos distribuídos para cada País.

Quadro 2 - Síntese dos principais resultados das regressões por País

\begin{tabular}{|c|c|c|c|c|c|c|c|}
\hline \multicolumn{2}{|c|}{ Variáveis/país } & ARG & BRA & CHL & COL & MEX & PER \\
\hline \multirow{2}{*}{ CONST. } & Coef. & 1,40 & $-6,11$ & 0,42 & $-15,3$ & 2,57 & 8,79 \\
\hline & Sig. & 0,774 & 0,411 & 0,908 & $0,093 * * *$ & $0,057 * * *$ & 0,178 \\
\hline \multirow{2}{*}{ TAM } & Coef. & $-0,19$ & 0,67 & $-0,00$ & 2,01 & $-0,20$ & $-0,89$ \\
\hline & Sig. & 0,741 & 0,398 & 0,996 & $0,047 * *$ & 0,129 & 0,244 \\
\hline \multirow{2}{*}{ INT.CAP } & Coef. & 0,40 & $-0,11$ & $-0,27$ & $-1,61$ & $-0,04$ & $-0,42$ \\
\hline & Sig. & 0,660 & 0,992 & 0,736 & 0,339 & 0,946 & 0,766 \\
\hline \multirow{2}{*}{ INT.INV } & Coef. & 2,39 & 4,48 & 0,56 & $-8,81$ & 0,01 & 0,33 \\
\hline & Sig. & 0,146 & 0,295 & 0,711 & 0,133 & 0,988 & 0,903 \\
\hline \multirow{2}{*}{ ALAV } & Coef. & 0,09 & $-0,00$ & $-0,00$ & $-0,69$ & $-0,01$ & $-0,11$ \\
\hline & Sig. & 0,020 ** & 0,880 & 0,965 & $0,015^{* *}$ & 0,337 & 0,699 \\
\hline \multirow{2}{*}{ ROA } & Coef. & 0,20 & 0,09 & $-0,33$ & $-0,04$ & $-4,96$ & $-7,16$ \\
\hline & Sig. & 0,747 & 0,902 & 0,651 & 0,857 & $0,001^{*}$ & $0,000^{*}$ \\
\hline \multicolumn{2}{|c|}{$\mathrm{R}^{2}$} & 0,043 & 0,002 & 0,001 & 0,077 & 0,046 & 0,062 \\
\hline \multicolumn{2}{|c|}{$\mathrm{F}$} & 0,161 & 0,863 & 0,992 & 0,055 & 0,005 & 0,004 \\
\hline \multicolumn{8}{|c|}{ Obs: $* 1 \%$ de significância; $* * 5 \%$ de significância; $* * * 10 \%$ significância. } \\
\hline \multicolumn{8}{|c|}{ Legenda: Argentina (ARG); Brasil (BRA); Chile (CHL); Colômbia (COL); México (MEX); Peru (PER). } \\
\hline
\end{tabular}

Fonte: Dados da pesquisa.

A análise realizada contemplou um total de 2.500 observações na América Latina, sendo 225 para a Argentina, 855 para o Brasil, 540 para o Chile, 190 para a Colômbia, 355 para o México e 335 para o Peru. Conforme verificado no Quadro 2, foi possível observar que o impacto das variáveis no modelo explicativo da ETR foi significativo na Argentina, Colômbia, México e Peru, sendo rejeitado acima de $86 \%$ o modelo brasileiro e acima de $99 \%$ o modelo Chileno.

Destaca-se que a Colômbia resultou no maior poder explicativo do modelo de regressão que foi de $7,7 \%$, de modo que o menor poder explicativo foi identificado no Chile e no Brasil, de 0,1 e 0,2 referente ao $\mathrm{R}^{2}$ do modelo, que respectivamente tiveram seus modelos rejeitados conforme destacado anteriormente (resultado de $\mathrm{F}$ ). A variação do $\mathrm{R}^{2}$ dos resultados dos modelos foi entre $4,3 \%$ e $7,7 \%$, excluídos os modelos rejeitados.

O baixo valor do poder explicativo dos modelos indica a existência de outros fatores que não foram investigados no modelo para explicar o que determina as taxas de imposto efetiva em cada País, uma vez que os resultados encontrados nesta pesquisa denotam diferenças existentes entre os países da América Latina.

Diante desses resultados, foi verificado que a variável tamanho (TAM) mostrou-se significativa ao nível de 5\% na Colômbia demonstrando um coeficiente positivo, sob o qual permite inferir que quanto maior o tamanho da empresa maior a taxa de imposto efetiva na realidade das empresas colombianas. Esta influência positiva entre o tamanho e a ETR corroboram com os achados dos estudos de Zimmerman (1983), Wang (1991), Omer, Molloy e Ziebart (1993), Calvé, Labatut e Molina (2005), Chen et al. (2010), Fernández-Rodríguez e Martinez-Arias (2011), Díaz, Fernandes-Rodrigues e Martinez-Arias (2011) e de FernándezRodríguez e Martínez-Arias (2014). Desta forma, aceita-se a hipótese $\mathrm{H}_{1}$ que remete a 
existência de uma influência positiva entre o tamanho e a taxa de imposto efetiva neste ambiente.

Entretanto, nos demais países, sendo a Argentina, Brasil, Chile, México e Peru, não foi encontrado significância estatística entre o tamanho da empresa e a ETR para determiná-la como variável explicativa da ETR. Assim, como os resultados encontrados nas pesquisas de Gupta e Newberry (1997), Wilkinson, Cahan e Jones (2001) e Liu e Cao (2007), que também não apresentaram significância, não é possível confirmar a influência do tamanho da empresa sobre a ETR nestes países no período analisado. Embora este resultado no contexto das empresas brasileiros confronta os resultados da pesquisa de Fernández-Rodríguez e MartínezArias (2014), que encontrou uma influencia positivamente significativa, no período analisado nesta pesquisa tais relacionamentos não foram confirmados. Desta forma o presente estudo rejeita a hipótese $\mathrm{H}_{1}$ que previa uma influência positiva do tamanho da empresa sobre a ETR para esse grupo de empresas dos países supracitados.

Em se tratando da variável que representa a intensidade de capital (INTCAP), os resultados não identificaram significância da variável em nenhum dos modelos de regressão utilizados para os países latino-americanos, dado que não permite confirmar a influência da variável sobre a taxa de imposto efetiva, com base nas observações realizada nas empresas argentinas, brasileiras, colombianas, chilenas, mexicanas e peruanas. Este resultado confronta os resultados atribuídos por Fernández-Rodríguez e Martínez-Arias (2014) que encontraram uma influência significativamente positiva para as empresas brasileiras e chinesas. Porém, ao mesmo tempo o presente estudo corrobora com o estudo de Fernández-Rodríguez e MartínezArias (2014) considerando que não foi encontrado significância para a variável intensidade de capital no contexto das empresas da Rússia e da Índia. Assim, o presente estudo resulta na rejeição da hipótese $\mathrm{H}_{2}$ que prevê a existência de uma influência positiva entre a intensidade de capital e a taxa de imposto efetiva.

Observando-se a variável intensidade de inventário (INTINV), verifica-se que esta também registrou a mesma rejeição que a variável de intensidade de capital, dado que o resultado do coeficiente atribuído para a intensidade de inventário não foi estatisticamente significativo para demonstrar a influência na taxa de imposto efetiva em todos os países da amostra (Argentina, Brasil, Colômbia, Chile, México e Peru). Estes resultados confrontam os resultados encontrados por Fernández-Rodríguez e Martínez-Arias (2014) que encontraram significância para a variável, implicando que quanto maior a intensidade de inventário maior é a carga fiscal das empresas brasileiras e chinesas, mais corroboram com os achados dos autores para o contexto das empresas da Rússia e da Índia que não apresentaram significância. Desta forma, os resultados desta esquisa destacam a rejeição da hipótese $\mathrm{H}_{3}$ que estabelecia a existência de uma influência positiva entre a intensidade de inventário e a taxa de imposto efetiva no contexto das empresas destes países.

No que tange à variável alavancagem (ALAV), os resultados no contexto das empresas argentinas apontaram significância ao nível 5\%, remetendo que quanto maior a alavancagem das empresas maior a ETR registrada pelas organizações. Assim, este resultado corrobora com os achados dos estudos de Harris e Feeny (2003), Janssen (2005), Chen et al. (2010) e Fernández-Rodríguez e Martínez-Arias (2011) que também apontaram uma influência positiva entre a alavancagem e a ETR, aceitando-se assim a hipótese $\mathrm{H}_{4}$ para este contexto, em virtude que estes resultados revelam que estas empresas buscam assumir mais dívidas para tentar reduzir sua carga tributária, assim como as empresas da China revelado no estudo de Fernández-Rodríguez e Martínez-Arias (2014). Entretanto, as empresas da Colômbia relataram outra realidade, de modo que os resultados evidenciaram uma influência negativa ao nível de 5\% de significância entre a alavancagem e a taxa de imposto efetiva, resultado que corrobora com os resultados encontrados nos estudos de Plesko (2003), Calvé, Labatut e Molina (2005), Liu e Cao (2007) e de Richardson e Lanis (2007), que também 
evidenciaram uma influência negativa entre a alavancagem e a ETR. Portanto, rejeita-se a hipótese $\mathrm{H}_{4}$ considerando a realidade das empresas colombianas.

No Brasil, Chile, México e Peru a variável que representa a alavancagem não registrou significância nos modelos, sob o qual este resultado não permite concluir a respeito da influência entre a alavancagem e a ETR, assim como os achados das pesquisas elaboradas por Kim e Limpaphayom (1998) e Wilkinson, Cahan e Jones (2001). Entretanto, este resultado confronta os achados de Fernández-Rodríguez e Martínez-Arias (2014) que encontraram uma influência negativamente significativa entre a alavancagem e a ETR das empresas do Brasil, Rússia e Índia. Desta forma, rejeita-se a hipótese $\mathrm{H}_{4}$ que apontava a existência de uma influência positiva entre a alavancagem e a taxa de imposto efetiva para as empresas que compõe a amostra analisada no contexto destes países.

Em relação a variável de rentabilidade, representada pelo retorno dos ativos (ROA), os resultados evidenciaram uma influência inversa entre as variáveis pesquisadas, indicando que quanto maior a rentabilidade menor a taxa de imposto efetiva no México e no Peru ao nível de $1 \%$ de significância. Assim sendo, a hipótese $\mathrm{H}_{5}$ foi rejeitada, tendo em vista que esperava-se uma influência positiva como destacada pelos estudos de Gupta e Newberry (1997), Plesko (2003), Calvé, Labatut e Molina (2005), Richardson e Lanis (2007), Chen et al. (2010) e de Fernández-Rodríguez e Martínez-Arias (2011). No entanto, a rejeição desta hipótese $\mathrm{H}_{5}$ no contexto das empresas mexicanas e peruanas corrobora com os achados do estudo de Derashid e Zhang (2003) que havia identificado para as empresas da Malásia uma influência negativa entre a rentabilidade e a ETR.

Denota-se ainda em relação a variável de rentabilidade que nos demais países analisados, compreendendo a Argentina, Brasil, Colômbia e Chile, os coeficientes registrados pelos modelos não foram estatisticamente significativos, não permitindo inferir a respeito da influência positiva ou negativa da rentabilidade sobre a taxa de imposto efetiva. Este resultado corrobora com o estudo realizado por Díaz, Fernandes-Rodrigues e Martinez-Arias (2011) e rejeita os achados do estudo de Fernández-Rodríguez e Martínez-Arias (2014) que encontrou para o Brasil um coeficiente positivamente significativo, assim como também para a China. Com base nesses resultados, rejeita-se a hipótese $\mathrm{H}_{5}$ que prevê a existência de uma influência positiva entre a rentabilidade e a taxa de imposto efetiva.

De modo geral, percebe-se conforme os resultados das hipóteses aceitas e rejeitadas na amostra selecionada para esta pesquisa, que cada País apresenta um contexto diferenciado, indicando que as determinantes da ETR variam de acordo com as singularidades e realidade de cada País individualmente, já que não percebeu-se nos dados aproximações em relação aos resultados encontrados para cada uma das variáveis utilizadas nos modelos de regressão de dados em painel. A Tabela 3 destaca uma síntese dos resultados evidenciados a partir das hipóteses testadas nesta pesquisa.

Tabela 3 - Resultados das hipóteses de pesquisa

\begin{tabular}{c|c|c|c}
\hline Hipótese & $\begin{array}{c}\text { Relação } \\
\text { esperada }\end{array}$ & Aceita no País & Rejeitada no País \\
\hline $\begin{array}{l}\text { H1 - Existe uma influência positiva entre o tamanho } \\
\text { e a taxa de imposto efetiva. }\end{array}$ & + & Colômbia. & $\begin{array}{c}\text { Argentina*; Brasil*; } \\
\text { Chile*; México*; Peru*. }\end{array}$ \\
\hline $\begin{array}{l}\text { H2 - Existe uma influência positiva entre a } \\
\text { intensidade de capital e a taxa de imposto efetiva. }\end{array}$ & + & Nada. & $\begin{array}{c}\text { Argentina*; Brasil*; } \\
\text { Colômbia*; Chile*; } \\
\text { México*; Peru*. }\end{array}$ \\
\hline $\begin{array}{l}\text { H3 - Existe uma influência positiva entre a } \\
\text { intensidade de inventário e a taxa de imposto efetiva. }\end{array}$ & + & Nada. & $\begin{array}{c}\text { Argentina*; Brasil*; } \\
\text { Colômbia*; Chile*; } \\
\text { México*; Peru*. }\end{array}$ \\
\hline $\begin{array}{l}\text { H4 - Existe uma influência positiva entre a } \\
\text { lavancagem e a taxa de imposto efetiva. }\end{array}$ & + & Argentina. & $\begin{array}{c}\text { Colômbia; Brasil*; } \\
\text { Chile*; México*; Peru*. }\end{array}$ \\
\hline
\end{tabular}




\begin{tabular}{l}
$\begin{array}{l}\text { H5 - Existe uma influência positiva entre a } \\
\text { rentabilidade e a taxa de imposto efetiva. }\end{array}$ \\
\hline OBS: * Não significativa no país analisado.
\end{tabular}

Fonte: Dados da pesquisa.

Em síntese, pode-se verificar que as determinantes da Taxa de Imposto Efetiva (ETR) diferem de País para País na América Latina, considerando o conjunto de observações realizadas e o período observado, o que estimula a realização de novos estudos, uma vez que os achados encontrados ao mesmo tempo que confirmam, também diferem de outros estudos realizados sobre a temática.

\section{Conclusões e Recomendações}

O estudo teve como objetivo verificar os fatores determinantes da taxa de imposto efetiva (ETR) de empresas da América Latina. Para tanto, desenvolveu-se uma pesquisa descritiva, com análise documental e abordagem quantitativa dos dados em uma amostra de 500 empresas dos países latino-americanos (Argentina; Brasil; Colômbia; Chile; México; Peru), no período de 2009 a 2013, perfazendo um total de 2.500 observações.

Os resultados encontrados evidenciam que o tamanho das empresas colombianas é fundamental para determinar a carga fiscal estimada pela taxa de imposto efetiva. A partir da análise dos dados constatou-se que quanto maior o tamanho destas empresas maior a ETR, implicando que as maiores organizações acabam pagando mais impostos, fato que demonstra e enfatiza a necessidade da utilização de um planejamento tributário adequado para atender os objetivos das organizações. Nos demais países analisados, os resultados não são conclusivos sobre a influência do tamanho da empresa na ETR.

Em se tratando das variáveis intensidade de capital (INTCAP) e intensidade de inventário (INTINV), os resultados encontrados não foram significativos no âmbito do modelo analisado. Desta forma, os achados deste estudo não apresentaram resultados conclusivos sobre a influência destas variáveis na carga fiscal dada pela ETR nos países analisados neste período.

No que tange a alavancagem, os resultados evidenciados na pesquisa indicam que para as empresas argentinas quanto maior a alavancagem maior a taxa de imposto efetiva, demonstrando que estas optam por assumir maiores dívidas para alavancar seu patrimônio e, neste País, acabam tendo que recolher mais impostos para o governo. No entanto, destaca-se que na Colômbia ocorre o inverso, sendo que as empresas que optam pelo aumento da alavancagem, através do aumento das dívidas de longo prazo, conseguem incorrer em menores taxas de imposto efetiva. Assim, os resultados permitem evidenciar que há fatores que podem estar relacionados a legislação de cada País para determinar a carga fiscal, tendo em vista que o endividamento pode estar sendo considerado na base de cálculo do valor para ser tributado. Já para os demais países, Brasil, Chile, México e Peru, os resultados não são conclusivos dado a não significância da variável no modelo de análise.

Quanto a rentabilidade, os resultados vão contra a maioria dos estudos encontrados na literatura, de modo que em nenhum dos modelos analisados observou-se a influência positiva salientada pelas pesquisas anteriores. Neste contexto, os resultados apontaram relação inversa para as empresas mexicanas e peruanas, demonstrando que quanto maior a rentabilidade destas organizações menor a carga fiscal incidente sobre a renda, o que sugere um melhor planejamento tributário adotado por estas empresas. Já em relação aos demais países, os resultados apresentados não foram conclusivos para inferir sobre a influência da rentabilidade sobre a ETR. 
De modo geral, considerando-se os resultados encontrados nesta pesquisa, pode-se concluir que existem diferenças entre os países analisados e suas determinantes da carga fiscal dada pela taxa de imposto efetiva, sob o qual não houve uma variável significativa com a mesma influência sobre a carga fiscal em todos os países que englobaram a análise da pesquisa. Desta forma, destaca-se que o estudo confronta os achados de FernándezRodríguez e Martínez-Arias (2014), no que tange a análise realizada para o contexto brasileiro, assim como também expande seus resultados analisando o contexto dos países latino-americanos, evidências estas que estimulam a realização de novos estudos.

Como limitações da pesquisa, destaca-se que a análise realizada foi generalizada, não observando-se eventuais diferenças institucionais, legais, econômicas e culturais entre os países da amostra selecionada para a realização do estudo, fatores estes que também podem impactar de alguma forma os resultados da pesquisa. Também não foram promovidas análises observando-se os diferentes setores de atividade a que as empresas da amostra investigada participam em cada País, assim como as características particulares que podem existir nas legislações aplicáveis nestes países, o que se apresenta como uma oportunidade de pesquisa para a realização de novos estudos.

Neste contexto, como recomendação para pesquisas futuras, sugere-se a realização de novos estudos com a segregação da amostra por setores de atividade, bem como a inclusão de variáveis de controle, como por exemplo o PIB de cada País, para que se possa capturar o efeito de possíveis diferenças econômicas que podem proporcionar impactos sobre os resultados das empresas. Adicionalmente, a aplicação de outras técnicas de análise estatística dos dados pode contribuir para a realização de novos estudos.

\section{Referências}

ASSAF NETO. Finanças corporativas e valor. 6. ed. São Paulo: Atlas, 2012.

CALDEIRA, Luciano Marques. Impactos dos tributos sobre a renda na geração de valor das empresas: um estudo comparativo internacional. 2006. 168 f. Dissertação (Mestrado em Administração) Programa de Pós-Graduação em Administração da Universidade de São Paulo, Ribeirão Preto, 2006.

CALVÉ PÉREZ, J. I.; LABATUT SERER, G.; MOLINA LLOPIS, R. Variables económicofinancieras que inciden sobre la presión fiscal soportada por las empresas de" reducida dimensión": Efectos de la Reforma Fiscal de 15 en las empresas de la Comunidad Valenciana. Revista Española de Financiación y Contabilidad, v. 34, n. 127, p. 875-897, 2005.

CAMPOS, L. H. Carga tributária no Brasil é a 2a maior da América Latina. Revista da Fiec, v. 81, n.8, 2014.

CANO, W. América Latina: notas sobre a crise. Economia e Sociedade, v. 18, n. 3, p. 603$621,2009$.

CAREY, D.; RABESONA, J. Average Effective Tax Rates on Capital, Labour and Consumption. Economics Department Working Papers - OECD, 2002.

CHEN, S., CHEN, X., CHENG, Q., \& SHEVLIN, T. Are family firms more tax aggressive than non-family firms?. Journal of Financial Economics, v. 95, n. 1, p. 41-61, 2010. 
DERASHID, C.; ZHANG, H. Effective tax rates and the "industrial policy" hypothesis: evidence from Malaysia. Journal of International Accounting, Auditing and Taxation, v. 12, n. 1, p. 45-62, 2003.

DÍAZ, A. R. F.; RODRÍGUEZ, E. F.; ARIAS, A. M. Factores condicionantes de la presión fiscal de las entidades de crédito españolas, $i$, existen diferencias entre bancos y cajas de ahorros?.Spanish Journal of Finance and Accounting/Revista Española de Financiación y Contabilidad, v. 40, n. 151, p. 491-516, 2011.

ESNOLDE, A. L.; GALlO, M. F.; PARISI, C.; PEREIRA, C. A. Percepção dos controllers em relação à gestão tributária. Anais... $9^{\circ}$ Congresso USP de Controladoria e Finanças. São Paulo - SP, 2009.

FEENY, S.; GILLMAN, M.; HARRIS, M. N. Econometric accounting of the Australian corporate tax rates: a firm panel example. Cardiff Economics Working Papers, 2005.

FERNÁNDEZ-RODRIGUEZ, E.; MARTINEZ-ARIASÃ, A. Determinants of effective Tax Rate: Evidence for USA and the EU. Intertax, v. 39, n. 8/9, p. 2011, 2011.

FERNÁNDEZ-RODRÍGUEZ, E.; MARTÍNEZ-ARIAS, A. Determinants of the Effective Tax Rate in the BRIC Countries. Emerging Markets Finance and Trade, v. 50, p. 214-228, 2014.

FORMIGONI, H.; ANTUNES, M. T. P.; PAULO, E. Diferença entre o Lucro Contábil e Lucro Tributável: Uma Análise sobre o Gerenciamento de Resultados Contábeis e Gerenciamento Tributário nas Companhias Abertas Brasileiras. BBR Brazilian Business Review, v. 6, n. 1, p. 44-61, 2009.

GIANNINI, S.; MAGGIULLI, C. The Effective tax rates in the EU Commission Studyon_corporate taxation: methodology calaspects, main results and policy implications. Paper prepared for the Conference "Corporate and Capital Income Taxation in the European Union" 2002.

GUPTA, S.; NEWBERRY, K. Determinants of the variability in corporate effective tax rates: Evidence from longitudinal data. Journal of Accounting and Public Policy, v. 16, n. 1, p. 134, 1997.

HARRIS, M. N.; FEENY, S. Habit persistence in effective tax rates. Applied Economics, v. 35, n. 8, p. 951-958, 2003.

JANSSEN, B. Corporate effective tax rates in the Netherlands. De Economist, v. 153, n. 1, p. 47-66, 2005.

KIM, K. A.; LIMPAPHAYOM, P. Taxes and firm size in Pacific-Basin emerging economies. Journal of International Accounting, Auditing and Taxation, v. 7, n. 1, p. 47$68,1998$.

KNIRSCH, D. Neutrality-based Effective Tax Rates. Tübinger Diskussionsbeiträge, n. 249, 2002. 
LAMMERSEN, L. The Measurement of Effective Tax Rates: Common Themes in Business Management and Economics. ZEW Discussion Papers, n. 2-46, 2002.

LIU, X.; CAO, S. Determinants of corporate effective tax rates: evidence from listed companies in China. Chinese Economy, v. 40, n. 6, p. 49-67, 2007.

MARKLE, K. S.; SHACKELFORD, D. A. Cross-country comparisons of corporate income taxes. National Bureau of Economic Research, 2011.

MINNICK, K.; NOGA, T. Do corporate governance characteristics influence tax management? Journal of Corporate Finance, v. 16, pp. 703-718, 2010.

OMER, T. C.; MOLLOY, K. H.; ZIEBART, D. A. An investigation of the firm sizeeffective tax rate relation in the 1980s. Journal of Accounting, Auditing \& Finance, v. 8, n. 2, p. 167-182, 1993.

PLESKO, G. A. An evaluation of alternative measures of corporate tax rates. Journal of Accounting and Economics, v. 35, n. 2, p. 201-226, 2003.

REGO, S. O. Tax-Avoidance Activities of U.S. Multinational Corporations. Contemporary Accounting Research, v. 20, n.4, p. 805-833, 2003.

RICHARDSON, G.; LANIS, R. Determinants of the variability in corporate effective tax rates and tax reform: Evidence from Australia. Journal of Accounting and Public Policy, v. 26, n.

6, p. 689-704, 2007.

SANTOS, A. R.; OLIVEIRA, R. C. M. Planejamento Tributário com ênfase em empresas optantes pelo lucro real. Anais... XVIII Congresso Nacional de Contabilidade, RS, Gramado. 2008.

SHACKELFORD, D. A.; SHEVLIN, T. Empirical tex research in accounting. Journal of Accounting and Economics, Rochester, v. 31, p. 321-387, 2001.

SHEVLIN, T. J. A Critique of Plesko's' An Evaluation of Alternative Measures of Corporate Tax Rates'. Available at SSRN 190436, 1999.

PAULO, E.; MARTINS, E.; CORRAR, L. J. Detecção do gerenciamento de resultados pela análise do diferimento tributário. RAE-Revista de Administração de Empresas, v. 47, n. 1, p. 46-59, 2007.

TANG, T. Y. H. Book-Tax Differences, a Proxy for Earnings Management and Tax Management - Empirical Evidence from China. 2005. Disponível em SSRN: <http://ssrn.com/abstract=872389>. Acesso em: 03 mar. 2014.

UTZIG, M. J. S.; MAGRO, C. B. D.; ZANELLA, G.; FREITAS, E. J. D.; DITTADI, J. R. Taxa de Imposto Efetiva sobre a renda nas empresas do Mercosul. Revista de Contabilidade do Mestrado em Ciências Contábeis da UERJ, v. 19, n. 2, p. 40-57, 2014. 
WANG, S-W. The relation between firm size and effective tax rates: A test of firms' political success. Accounting Review, p. 158-169, 1991.

WILKINSON, B. R.; CAHAN, S. F.; JONES, G. Strategies and dividend imputation: the effect of foreign and domestic ownership on average effective tax rates. Journal of International Accounting, Auditing and Taxation, v. 10, n. 2, p. 157-175, 2001.

ZIMMERMAN, J. L. Taxes and firm size. Journal of Accounting and Economics, v. 5, p. 119-149, 1983. 